\title{
Palliation of Dysphagia in Locally Advanced Carcinoma Esophagus- Exploration of Two Different Radiotherapy Schedules
}

\author{
${ }^{1}$ Sheeba Bhardwaj (Junior Resident, Radiation Oncology, PGIMS, Rohtak) \\ ${ }^{2}$ Rakesh Dhankhar (Senior Professor, Radiation Oncology, PGIMS, Rohtak) \\ ${ }^{3}$ Vivek Kaushal (Senior Professor and Head of Department, Radiation Oncology, PGIMS, Rohtak) \\ ${ }^{4}$ Rajeev Atri (Professor, Radiation Oncology, PGIMS, Rohtak) \\ ${ }^{5}$ Anil Kumar Dhull (Assistant Professor, Radiation Oncology, PGIMS, Rohtak) \\ ${ }^{6}$ Peoli Mukutawat (Junior Resident, Radiation Oncology, PGIMS, Rohtak) \\ ${ }^{7}$ Shweta Bhardwaj (Junior Resident, Anaesthesiology, PGIMS, Rohtak) \\ Department of Radiation Oncology, Pandit B.D. Sharma PGIMS, Rohtak
}

\begin{abstract}
$>$ Introduction-

Esophageal cancer is the seventh most common cancer constituting $3.2 \%$ of all cancer cases and sixth most common cause of mortality constituting $\mathbf{5 . 3 \%}$ cases worldwide in 2018. Most common presenting symptom is dysphagia which is seen in $80-90 \%$ of patients. Most patients present in locally advanced stage and because of extensive local disease palliative radiotherapy plays a significant role.
\end{abstract}

\section{$>$ Aim and objectives-}

Aim of the study was to compare two palliative radiotherapy schedules- 30 Gy in 10 fractions over 2weeks versus $20 \mathrm{~Gy}$ in 5 fractions over 1-week in locally advanced carcinoma esophagus. Objectives were to compare above schedules based upon symptomatic relief and tolerability by the patient.

\section{$>$ Material and methods-}

The study was a randomized control study done from July 2017 - December 2018 on 60 previously untreated, histo-pathologically proven patients of squamous cell carcinoma esophagus (locally advanced) reporting in the Department of Radiation oncology, Pandit B. D. Sharma PGIMS, Rohtak, where palliative radiotherapy was indicated as the treatment. Patients were divided into two groups of 30 patients each; Study group received- $30 \mathrm{~Gy}$ in 10 fractions over 2-weeks and Control group received $20 \mathrm{~Gy}$ in 5 fractions over 1week. The symptomatic relief was assessed according to dysphagia score at 1- month after completion of radiation treatment. A reduction of at least 1 point in dysphagia score was depicted as improvement of dysphagia.

\section{$>$ Observations and result-}

Dysphagia was the most common presenting symptom seen in all the patients followed by chest pain and weight loss. $80 \%$ patients in study group and $76.6 \%$ patients in control group showed improvement in dysphagia. $25 \%$ patients in study group and $43.4 \%$ in control group developed recurrent dysphagia. The mean duration of development of recurrent dysphagia in study group was 2.5 months and 5.6 months in control group. It was concluded that both the radiotherapy schedules were comparable in providing symptomatic relief; both radiotherapy schedules were tolerable by the patients.

\section{INTRODUCTION}

Esophageal cancer is the seventh most common cancer constituting $3.2 \%$ of all cancer cases and sixth most common cause of mortality constituting $5.3 \%$ cases worldwide in 2018 ${ }^{1}$. Major risk factors for esophageal cancer development are tobacco and alcohol abuse; risk of SCC decreases substantially after smoking cessation whereas the risk for adenocarcinoma remains unchanged even after smoking cessation. Obesity, high BMI (Body Mass Index), GERD (Gastroesophageal reflux disease) and Barrett's esophagus are also strong risk factors for adenocarcinoma of the esophagus. ${ }^{2,3}$ Dysphagia is the most common complaint in $80-90 \%$ of patients and as the disease progresses it leads to nutritional compromise, pain, reduced performance status and deterioration of quality of life. ${ }^{4}$ Therefore, the main aim of treatment in patients with incurable esophageal cancer is palliation of dysphagia. ${ }^{5}$ Many of these patients are not fit enough for surgical resection or curative radiochemotherapy. ${ }^{6}$ Therefore, palliation remains an important goal. ${ }^{7}$ The present study was designed to evaluate symptomatic relief in patients with locally advanced esophageal carcinoma who received two different schedules of palliative radiotherapy.

\section{MATERIAL AND METHODS}

It was a randomized control study done from July 2017 to December 2018 on 60 previously untreated, histopathologically proven patients of squamous cell carcinoma esophagus reporting in the Department of Radiation oncology, Pt. B. D. Sharma PGIMS, Rohtak; where palliative radiotherapy was indicated as the treatment. Inclusion criteria for the study were patients having locally advanced carcinoma esophagus, inoperable tumor or who were unfit for surgery and other combination chemotherapy treatment modality and where palliative radiotherapy was 
indicated, Karnofsky Performance Status $\geq 60$, normal complete hemogram, renal function tests and liver function tests and patients who signed the informed consent and were ready to be on follow up for 6 months after completion of treatment. Patients were randomized into two groups of 30 patients each; study group received- $30 \mathrm{~Gy}$ in 10 fractions over 2-weeks and control group received- 20
Gy in 5 fractions over 1-week. The symptomatic relief was assessed at 1- month after completion of radiation treatment according to dysphagia score (table-1). Improvement of dysphagia was defined as a decrease of at least 1 point in dysphagia score. All the patients were followed up in OPD for a period of at least 6 months.

\begin{tabular}{|c|c|}
\hline Score & Swallowing status \\
\hline 0 & Asymptomatic \\
\hline 1 & Can eat solid diet with some dysphagia \\
\hline 2 & Eats semi-solid diet \\
\hline 3 & Can take liquid diet \\
\hline 4 & Complete dysphagiaj \\
\hline
\end{tabular}

Table 1:- Dysphagia score

\section{OBSERVATIONS AND RESULT-}

Table 2 shows improvement in dysphagia score post palliative radiotherapy; observations were made at one month after completion of radiation treatment. $80 \%$ patients in study group and $76.6 \%$ patients in control group showed improvement in dysphagia score while $20 \%$ patients in study group and $23.3 \%$ patients in control group showed no reduction in dysphagia score. Overall $78.3 \%$ patients showed improvement in dysphagia after palliative radiotherapy.

\begin{tabular}{|c|c|c|c|}
\hline Improvement in dysphagia score & $\begin{array}{c}\text { Study group } \\
\mathrm{n}=30\end{array}$ & $\begin{array}{c}\text { Control group } \\
\mathrm{n}=30\end{array}$ & $\mathrm{p}$ value \\
\hline Yes (\%) & $24(80 \%)$ & $23(76.6 \%)$ & 0.17 \\
\hline No (\%) & $6(20 \%)$ & $7(23.3 \%)$ \\
\hline
\end{tabular}

Table 2:- Dysphagia score improvement post radiotherapy

In study group 25\% (n=24) patients developed recurrent dysphagia and in control group $43.4 \% \quad(n=23)$ patients developed recurrent dysphagia. The mean duration of development of recurrent dysphagia in study group was 2.5 months and in control group it was 5.6 months. The p value was 0.11 which was statistically non- significant.

\section{DISCUSSION}

This prospective study was conducted on 60 previously untreated, histo-pathologically proven patients of Carcinoma esophagus reporting in the Department of Radiotherapy, Pandit B. D. Sharma PGIMS, Rohtak, where palliative radiotherapy was indicated as the treatment. Patients were divided into two groups of 30 patients each; study group received- 30 Gy in 10 fractions over 2-weeks and control group received $20 \mathrm{~Gy}$ in 5 fractions over 1 week. The symptomatic relief was assessed at 1- month after completion of radiation treatment according to dysphagia score. A reduction of at least 1 point in dysphagia score was depicted as improvement of dysphagia.

All the patients had complaint of dysphagia. Second most common symptom was chest pain seen followed by weight loss. Squamous cell carcinoma was the histology in both groups with moderately differentiated subtype being the commonest.

Out of all the patients $(n=60) 78.3 \%$ patients $(80 \%$ in study group and $76.6 \%$ in control group) patient showed reduction in dysphagia score while $21.7 \%$ patients $(20 \%$ in study group and $23.3 \%$ in control group) showed no reduction in dysphagia score. $25 \%$ patients in study group and $43.4 \%$ in control group developed recurrent dysphagia. The mean duration of development of recurrent dysphagia in study group was 2.5 months and in control group it was 5.6 months. The mean duration of follow up was 6.9 months in study group and 6.6 months in control group.

\section{CONCLUSION}

It was concluded that both the radiotherapy schedules were comparable in providing symptomatic relief; and were tolerable by the patients. The development of recurrent dysphagia was more common in control group thereby requiring secondary interventions; however the results were statistically non-significant. Radiotherapy in locally advanced cases of carcinoma esophagus is an effective modality for palliation of dysphagia. 


\section{REFERENCES}

[1]. Global Cancer Statistics 2018: GLOBOCAN Estimates of Incidence and Mortality Worldwide for 36 Cancers in 185 Countries. Cancer J Clin. 2018;68:394-424.

[2]. Vaughan TL, Davis S, Kristal A, Thomas DB. Obesity, alcohol, and tobacco as risk factors for cancers of the esophagus and gastric cardia: adenocarcinoma versus squamous cell carcinoma. Cancer Epidemiol Biomarkers Prev. 1995;4:85-92.

[3]. Cossentino MJ, Wong RK. Barrett's esophagus and risk of esophageal Adenocarcinoma. Semin Gastrointest Dis. 2003;14:128-35.

[4]. Cohen DJ, Leichman L. Controversies in the treatment of local and locally advanced gastric and esophageal cancers. J Clin Oncol. 2015;33:1754-9.

[5]. Watt E, Whyte F. The experience of dysphagia and its effect on the quality of life of patients with oesophageal cancer. Eur J Cancer care. 2012;2:18393.

[6]. Ordu AD, Nieder C, Geinitz H. Radio/chemo therapy for locally advanced squamous cell carcinoma of the esophagus: long-term outcome. Strahlenther Onkol. 2015;191:153-60.

[7]. Weigel TL, Frumiento C, Gaumintz E. Endoluminal palliation for dysphagia secondary to esophageal carcinoma. Surg Clin Am. 1982(4):747-61. 